\title{
Electronic
Interfaces
}

\section{Zachary Porter}

Office of Science, Science Undergraduate Laboratóry Internship (SULI) Program

This work was supported in part by the U.S. Department of Energy, Office of Science, Office of Workforce Development for Teachers and Scientists (WDTS) under the Science Undergraduate Laboratory Internship (SULI) program, under Contract No. DE-AC02-76SF00515. 


\section{Electronic Structure of Buried Interfaces}

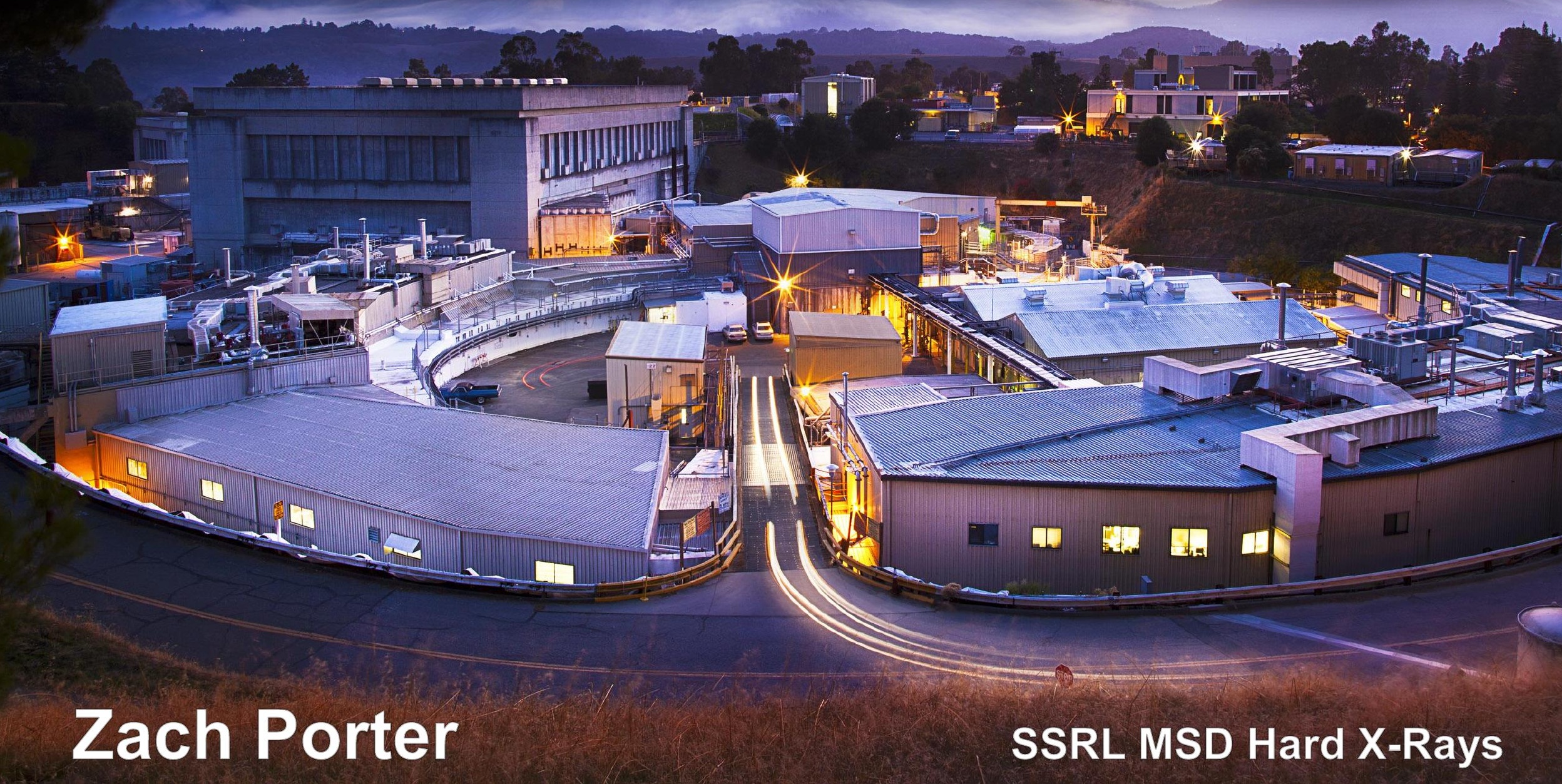




\section{Abstract}

In the electronics behind computer memory storage, the speed and size are dictated by the performance of permanent magnets inside devices called read heads. Complicated magnets made of stacked layers of thin films can be engineered to have properties that yield more energy storage and faster switching times compared to conventional iron or cobalt magnets. The reason is that magnetism is a result of subtle interactions amongst electrons; just how neurons come together on large scales to make cat brains and dog brains, ensembles of electrons interact and become ferromagnets and paramagnets. These interactions make magnets too difficult to study in their entirety, so I focus on the interfaces between layers, which are responsible for the coupling materials physicists hope to exploit to produce next-generation magnets.

This project, I study a transition metal oxide material called LSCO, Lanthanum Cobaltite, which can be a paramagnet or a ferromagnet depending on how you tweak the electronic structure. It exhibits an exciting behavior: its sum is greater than the sum of its parts. When another similar material called a LSMO, Lanthanum Manganite, is grown on top of it, their interface has a different type of magnetism from the LSCO or the LSMO! I hope to explain this by demonstrating differently charged ions in the interface. The typical method for quantifying this is $\mathrm{x}$-ray absorption, but all conventional techniques look at every layer simultaneously, averaging the interfaces and the LSCO layers that we want to characterize separately. Instead, I must use a new reflectivity technique, which tracks the intensity of reflected $\mathrm{x}$-rays at different angles, at energies near the absorption peaks of certain elements, to track changes in the electronic structure of the material. The samples were grown by collaborators at the Takamura group at U.C. Davis and probed with this "resonant reflectivity" technique on Beamline 2-1 at the Stanford Synchrotron Radiation Lightsource. This project was funded by the Department of Energy and supported by the SLAC National Accelerator Laboratory.

Preliminary results suggest that different ionic charges are indeed responsible for the different magnetic properties at the interface, which is promising because charge is easy to control. Once scientists understand how to tune the magnetic properties of materials like LSCO and LSMO, industries get closer to designing the magnets that will soon revolutionize consumer electronics. 


\section{Magnets}

SLAAC
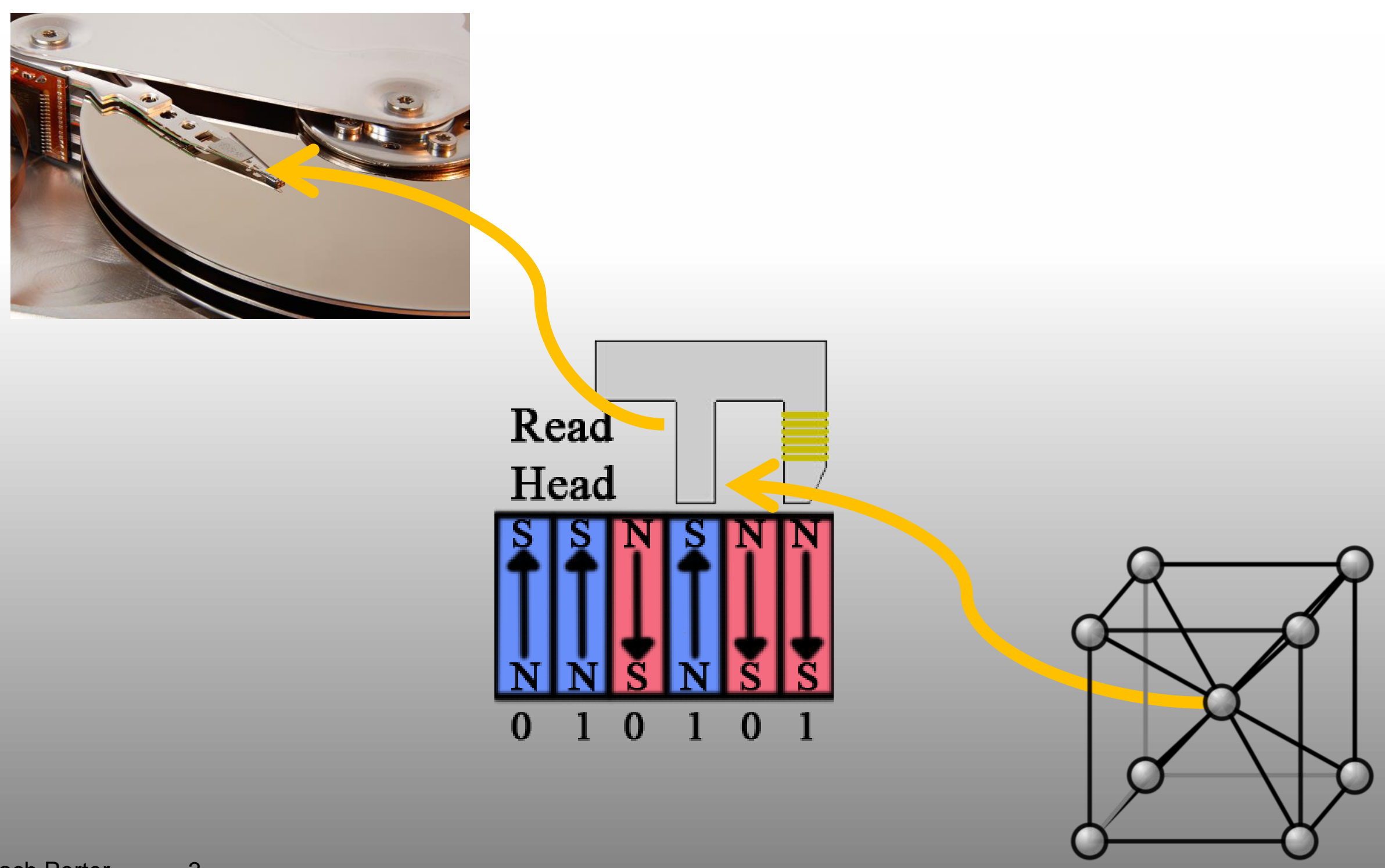


\section{Emergent magnetic properties}

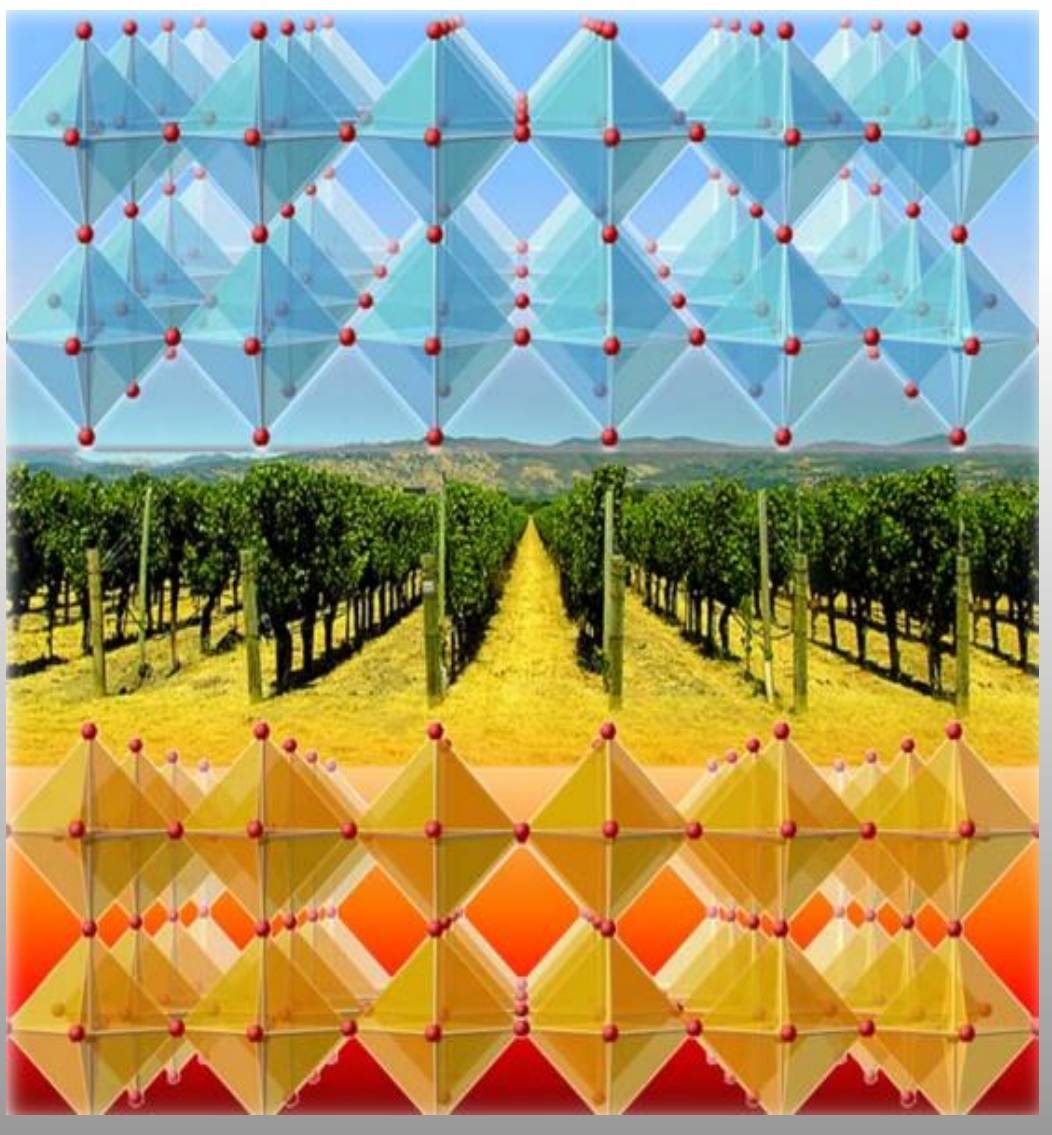

Zach Porter

\author{
LSMO \\ $\left(\mathrm{La}_{0.7} \mathrm{Sr}_{0.3} \mathrm{MnO}_{3}\right)$ \\ Interface \\ LSFO \\ $\left(\mathrm{La}_{0.7} \mathrm{Sr}_{0.3} \mathrm{FeO}_{3}\right)$
}




\section{Outline}

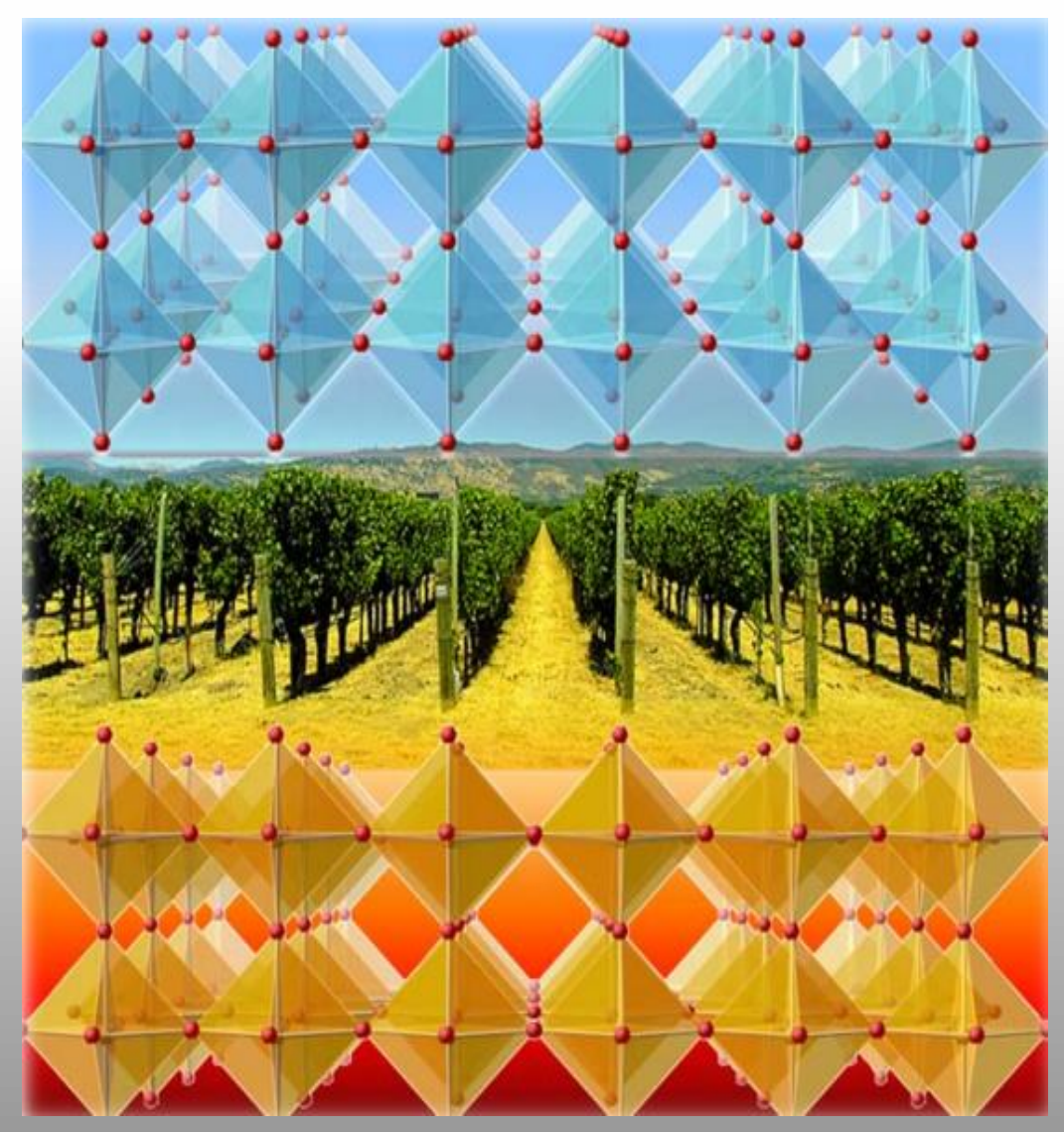

\section{Absorption (what I want)}

\section{Reflectivity \\ (to get absorption)}

\section{Electronic Properties}




\section{X-ray absorption spectroscopy}

SLLAC
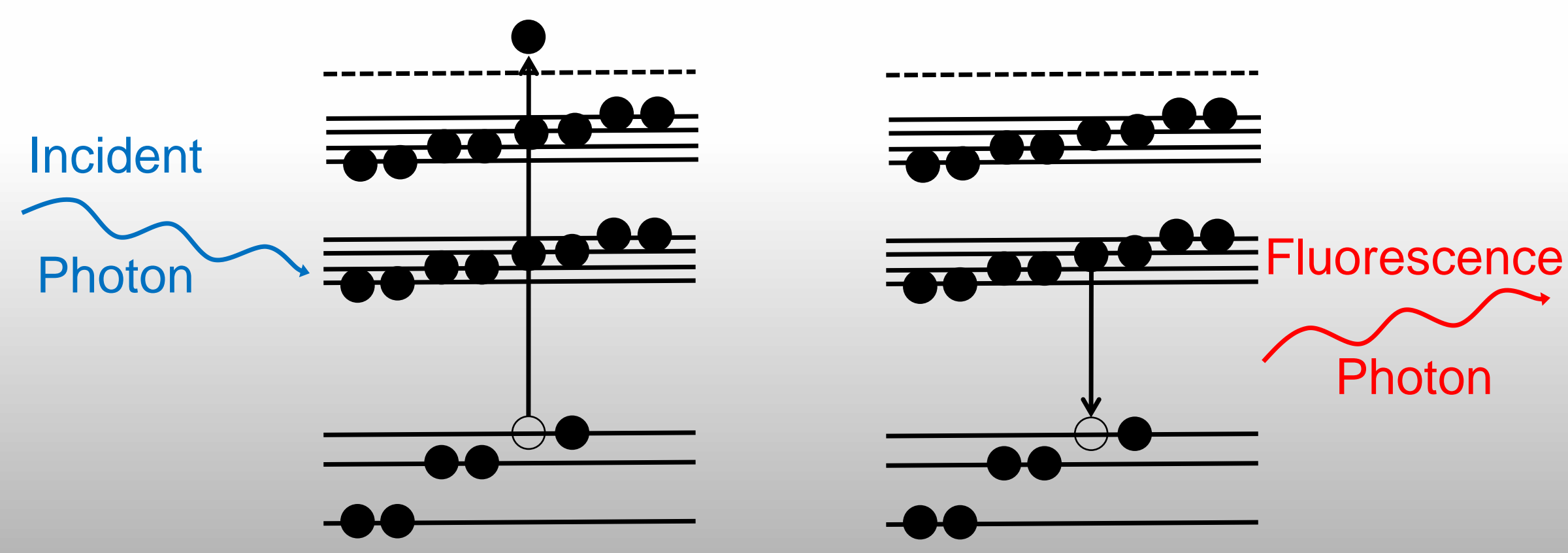


\section{X-ray absorption spectroscopy}

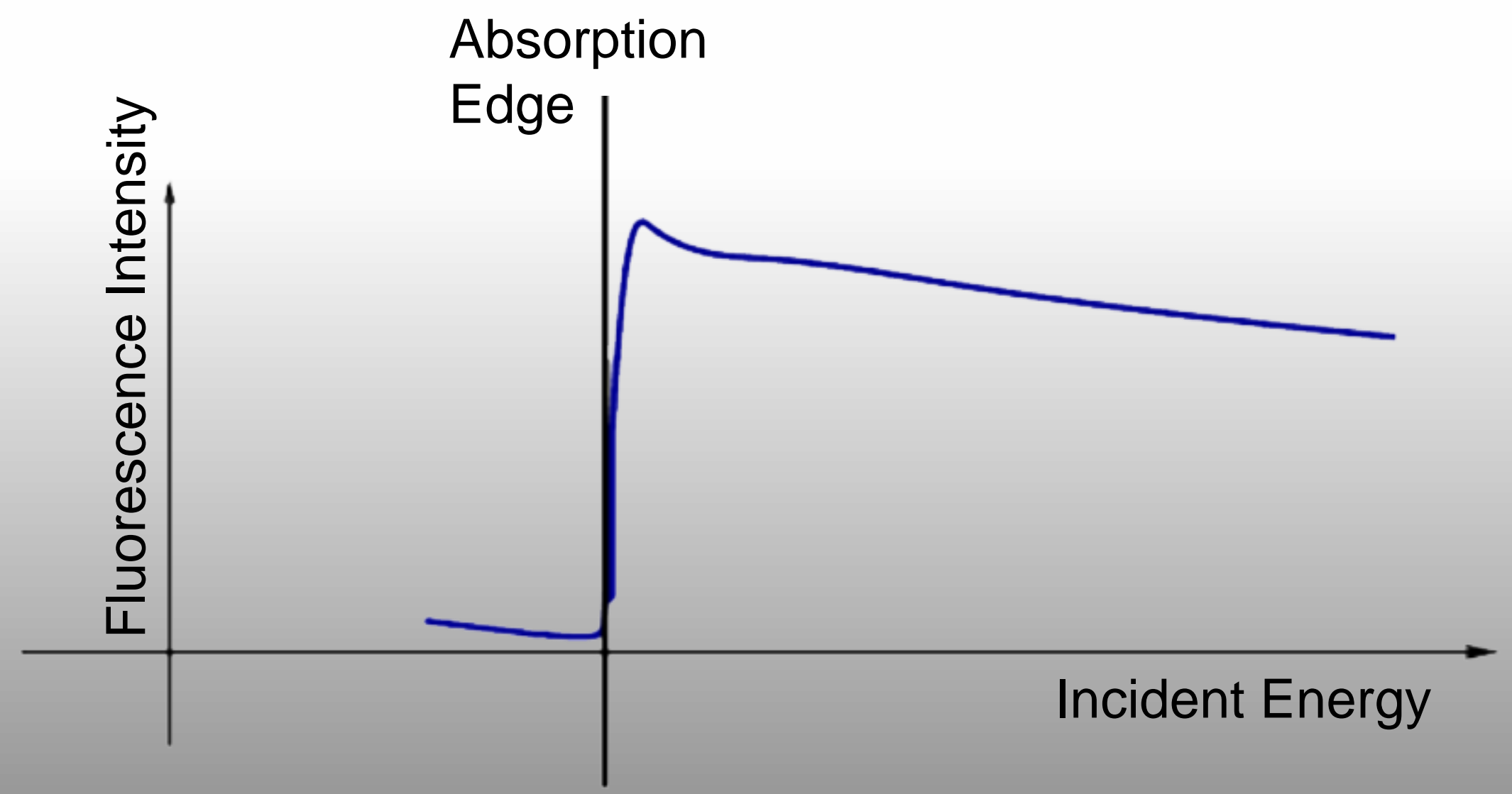




\section{X-ray absorption for $\mathrm{e}^{-}$structure}

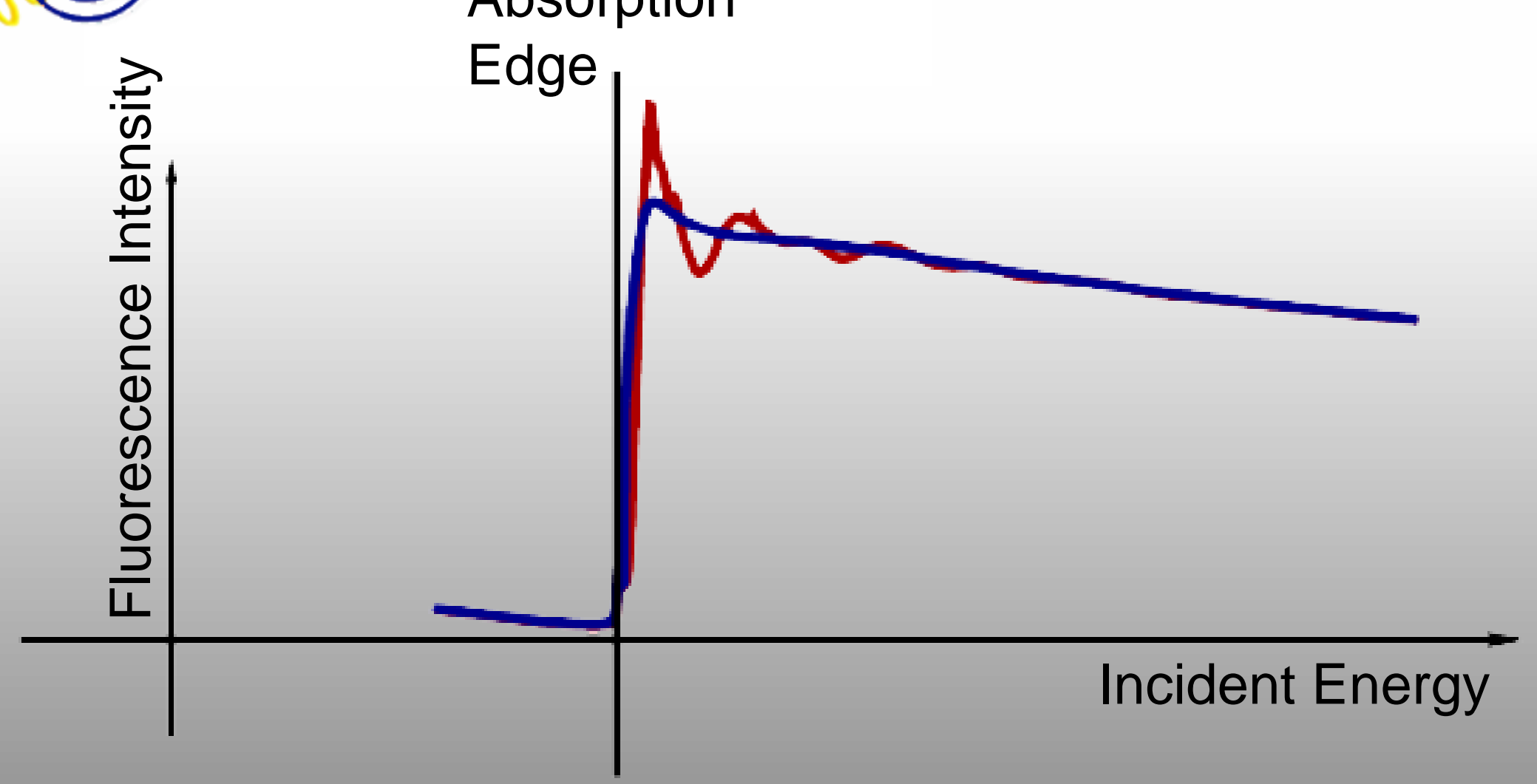




\section{X-ray absorption for $\mathrm{e}^{-}$structure}

SLACOC

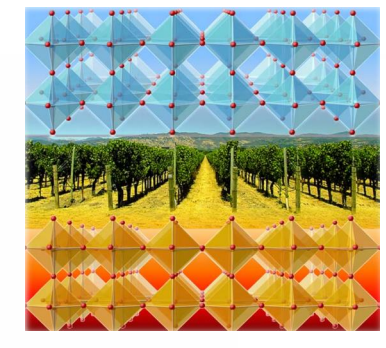

$\mathrm{Mn} \mathrm{K}_{\beta}$

Edge

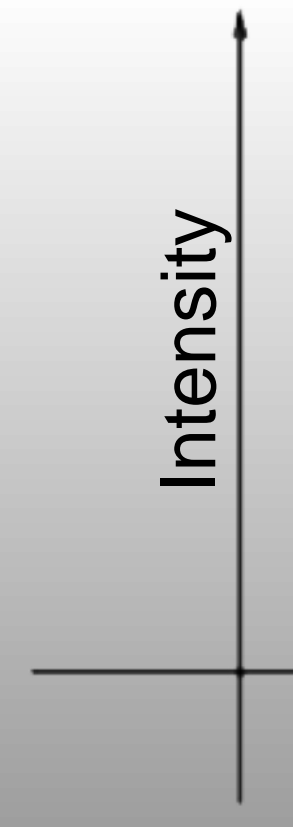

\section{Incident Energy}




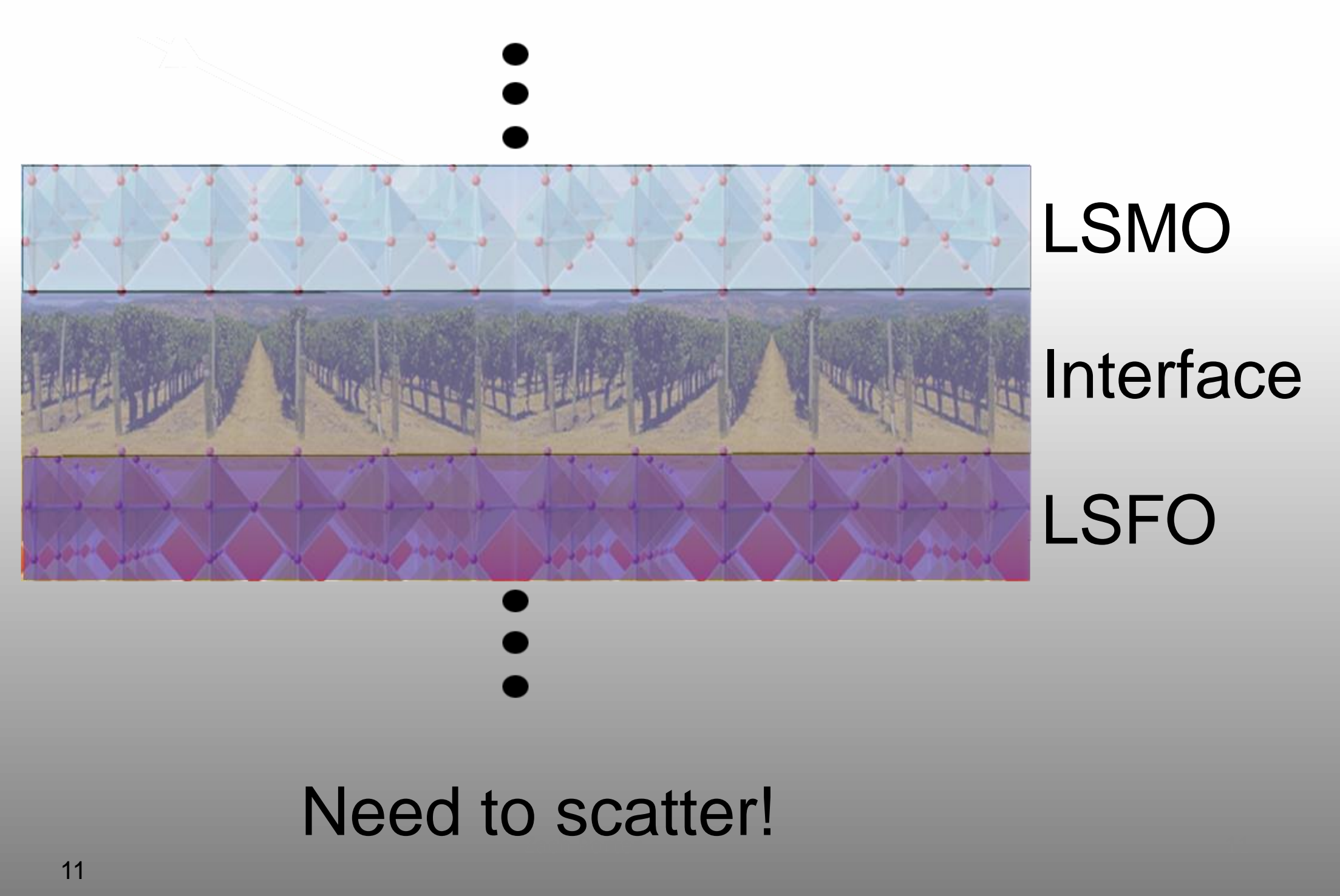




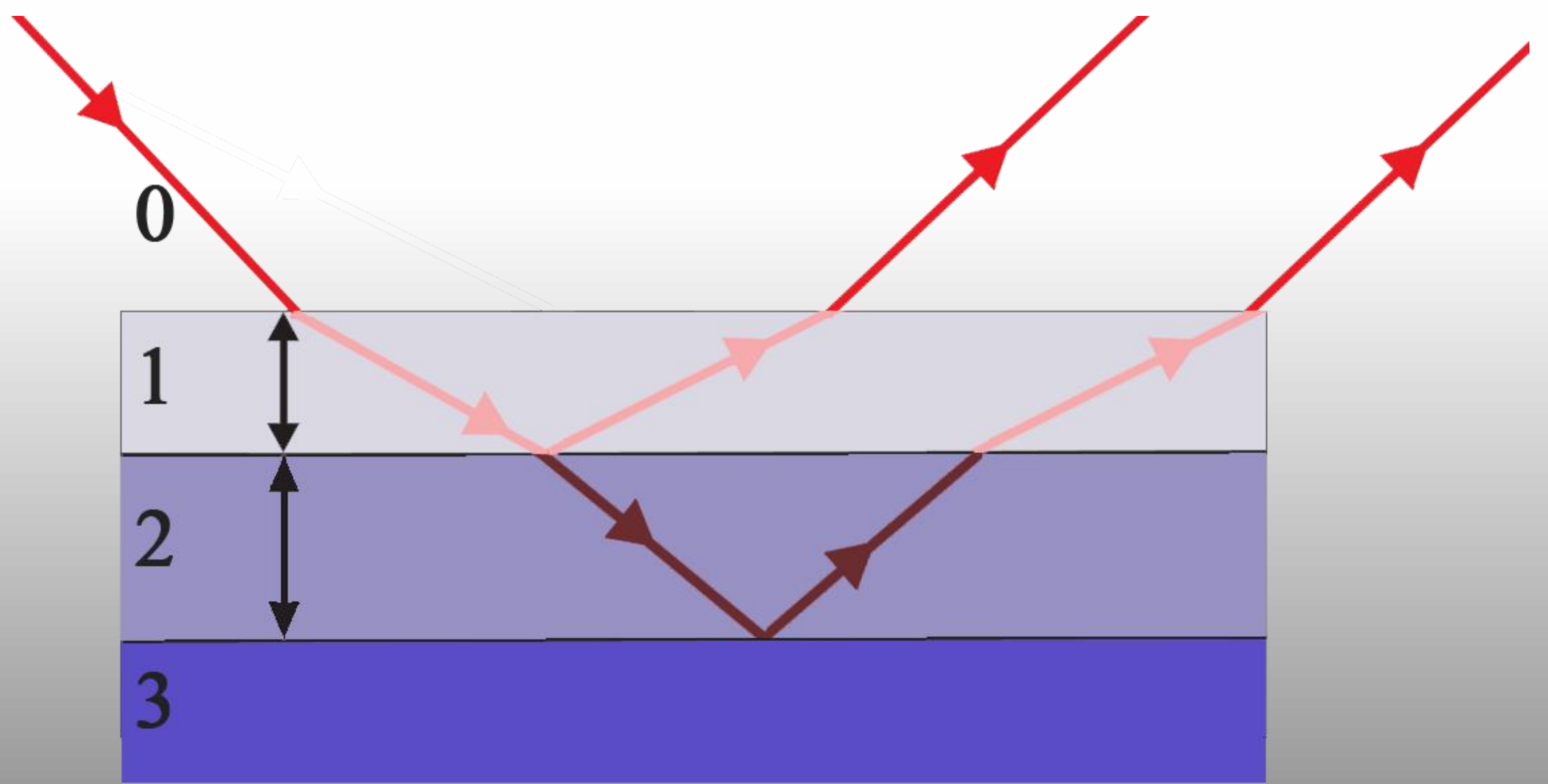




\section{Beamline 2-1}

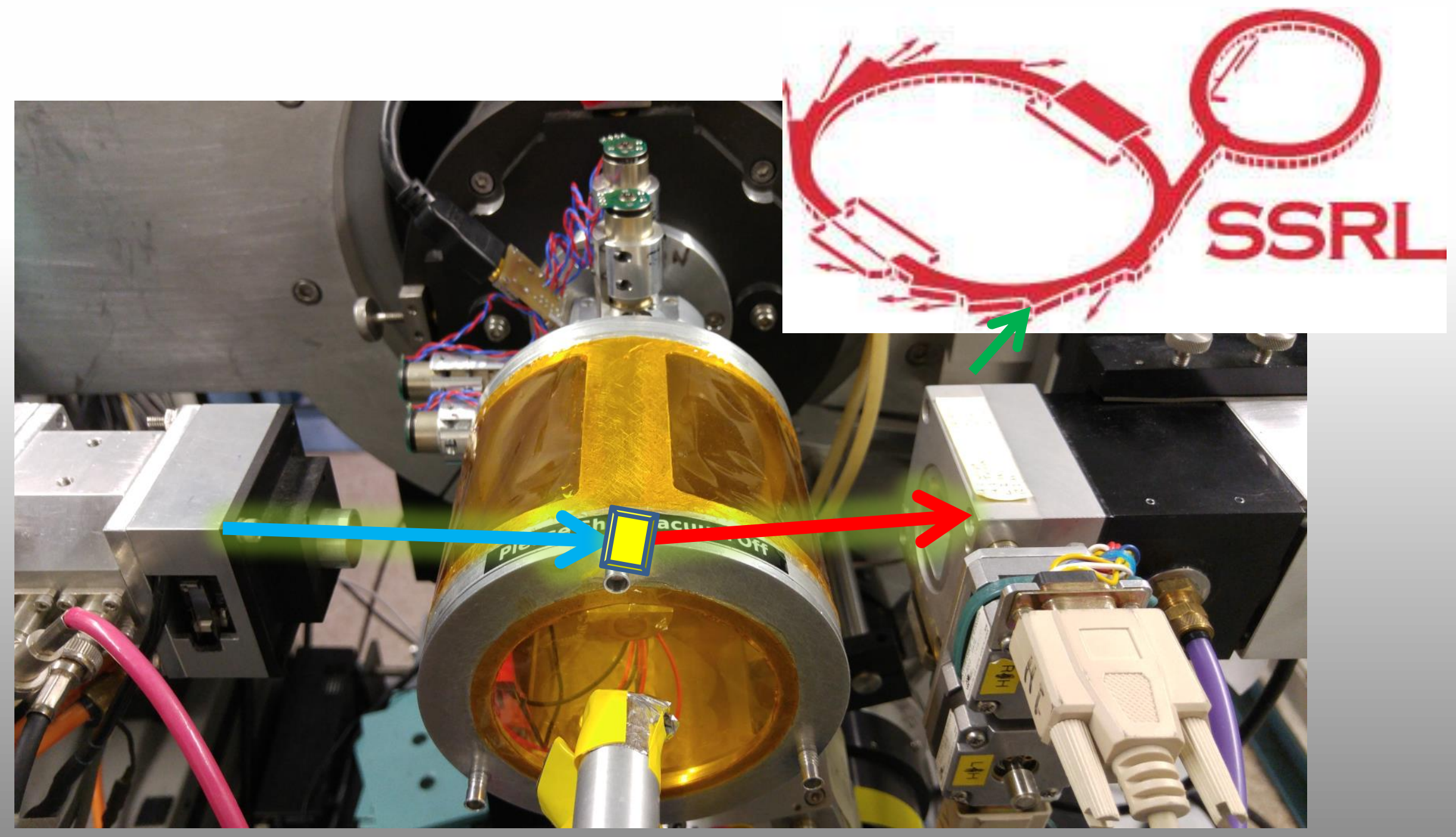




\section{Reflectivity}

SLLAC

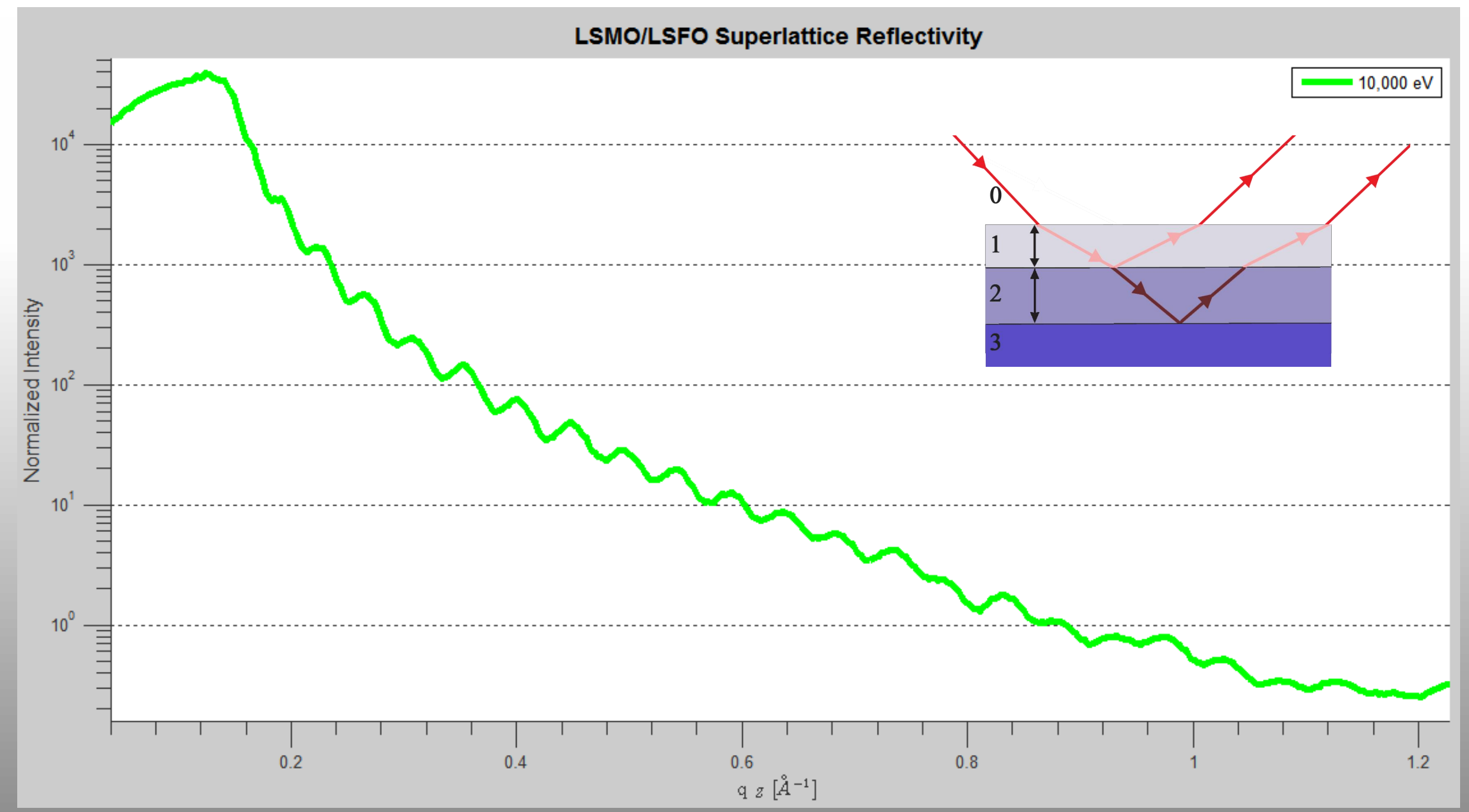




\section{Resonant reflections}

- Increase contrast with absorption

- Change in interference pattern

- See what oxidation states are

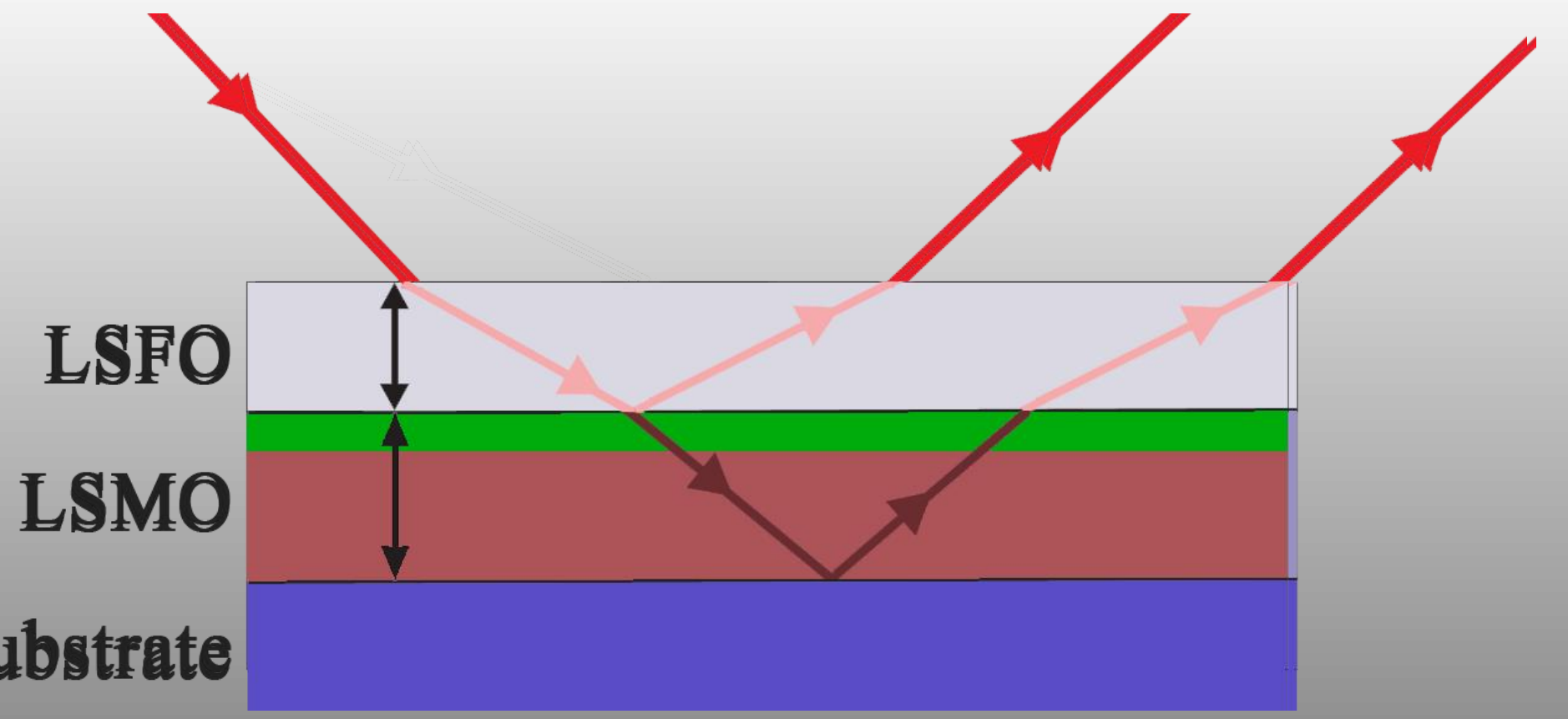




\section{Resonant reflectivity}

SL_A

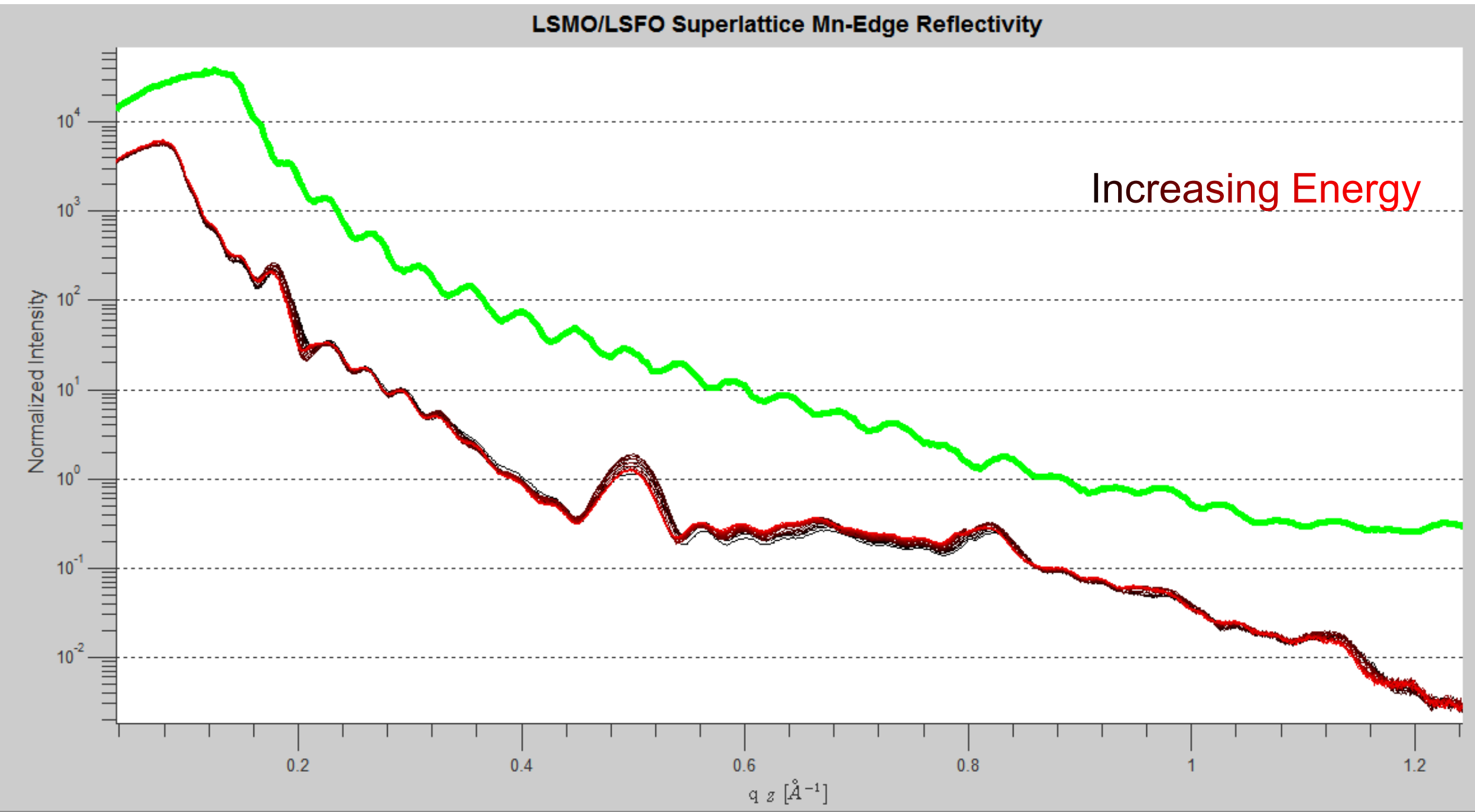




\section{Resonant reflectivity seems valid}
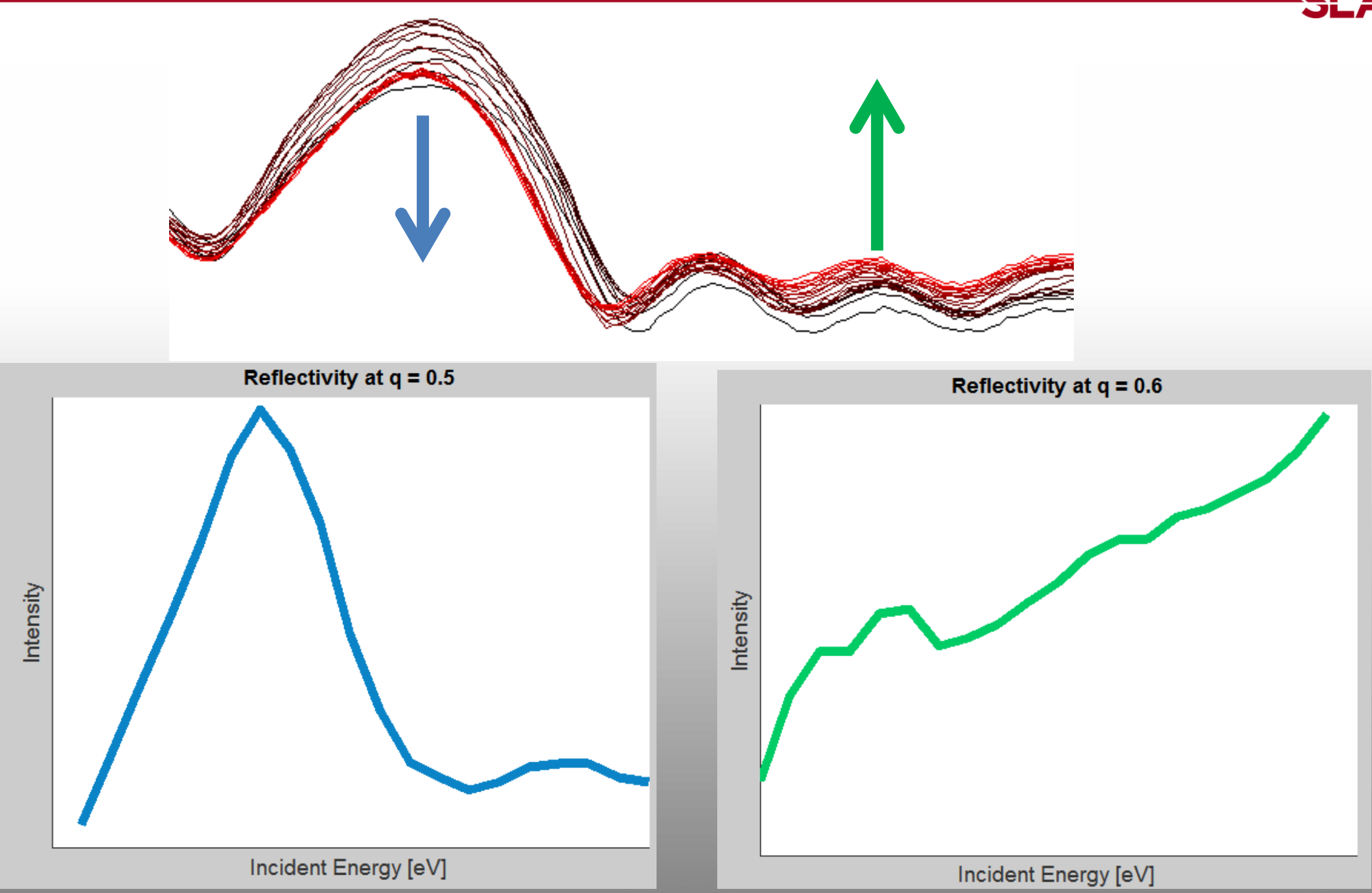


\section{Electronic structure}

SELAO

\section{Fitting reflectivity curves}

results are pending

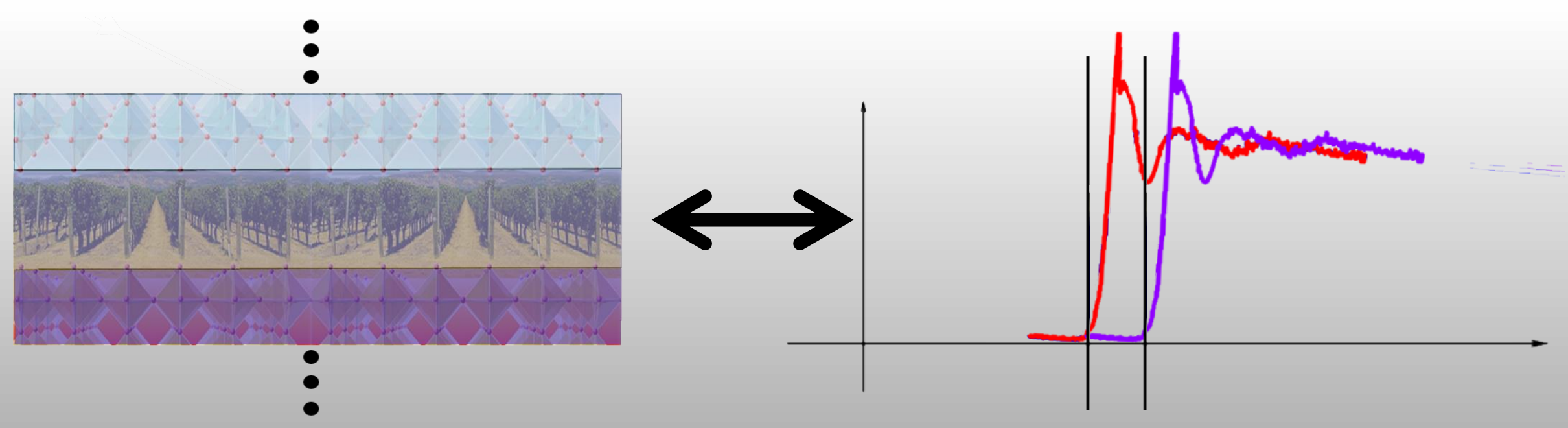

Back-convert to absorption

characterize interfaces! 


\section{Kramers-Kronig transforms}

SLAAC

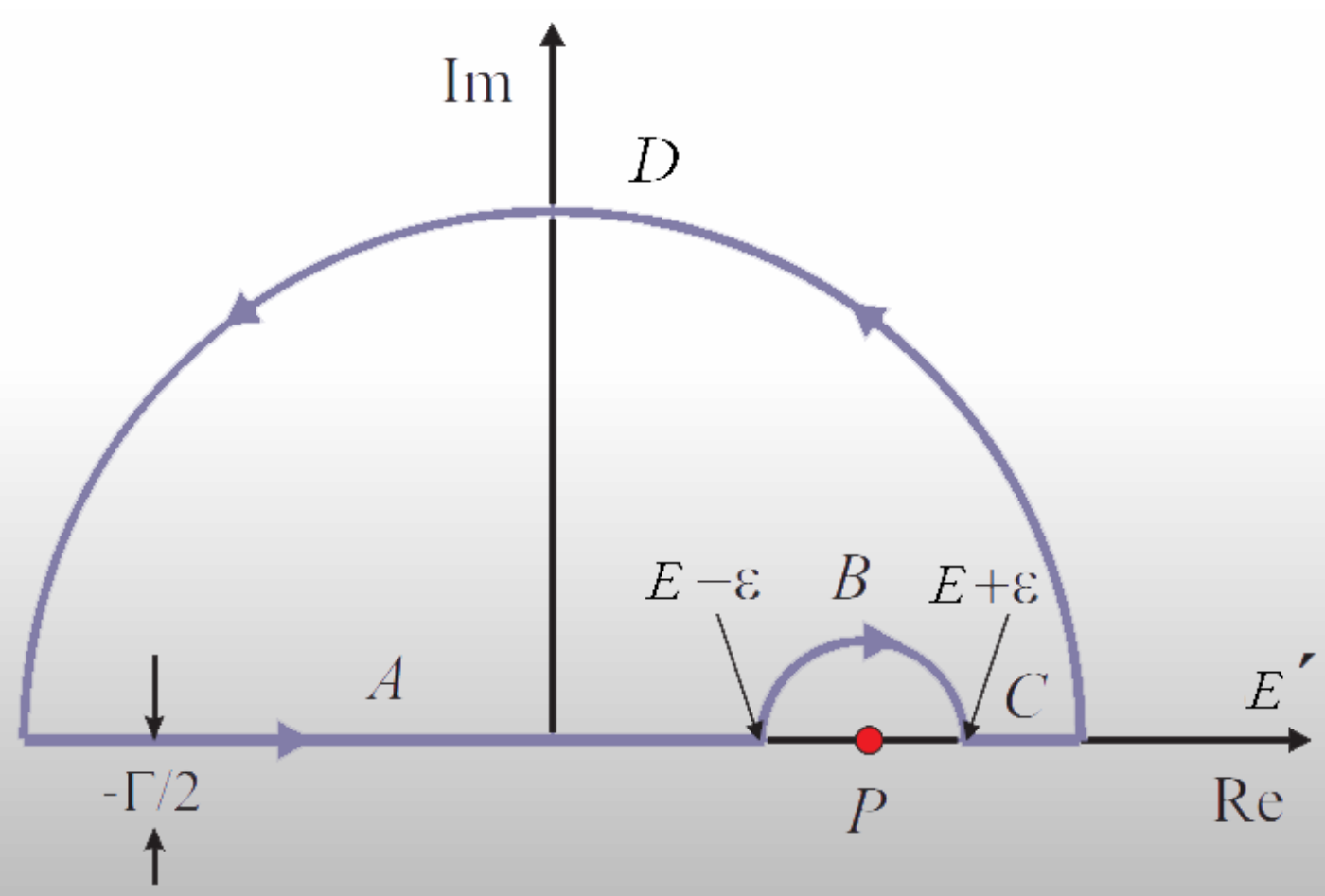

$$
f^{\prime}(E)+i f^{\prime \prime}(E):=\frac{1}{i \pi} \mathcal{P} \int_{-\infty}^{\infty} \frac{f^{\prime}\left(E^{\prime}\right)+i f^{\prime \prime}\left(E^{\prime}\right)}{E^{\prime}-E} d E^{\prime}
$$




\section{THE method for buried interfaces}

SLLAO

Understand coupling across layers
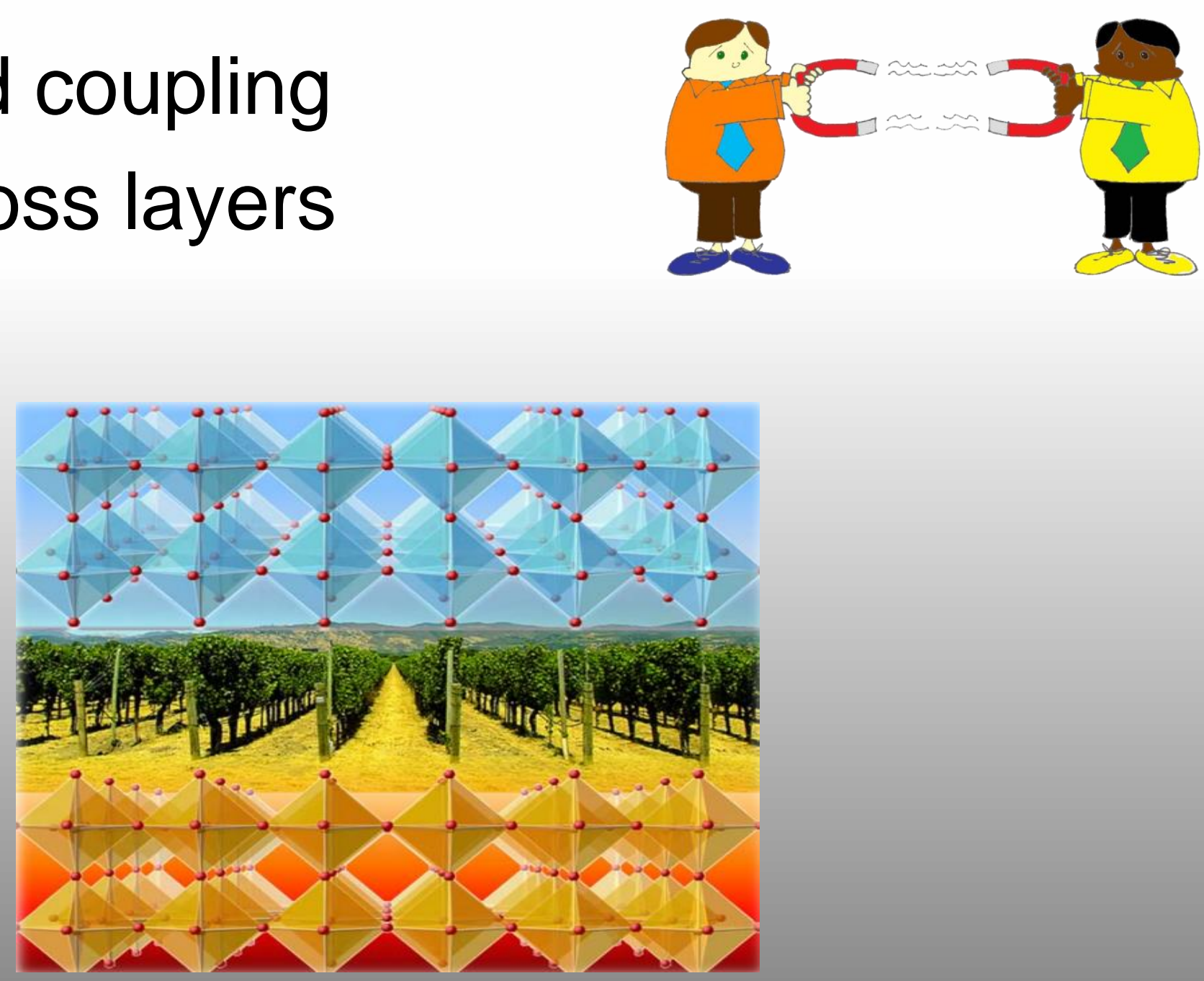


\section{Special Thanks}

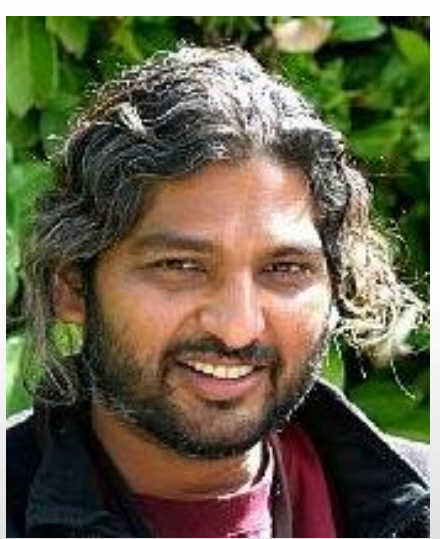

Apurva Mehta

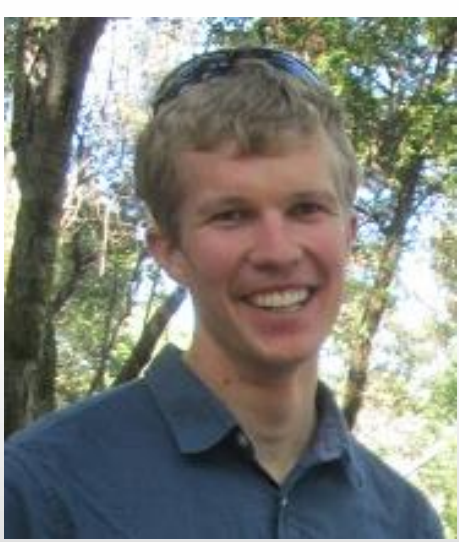

Trevor Petach

Takamura Group @ UC Davis

Beamline Engineers Duty Operators

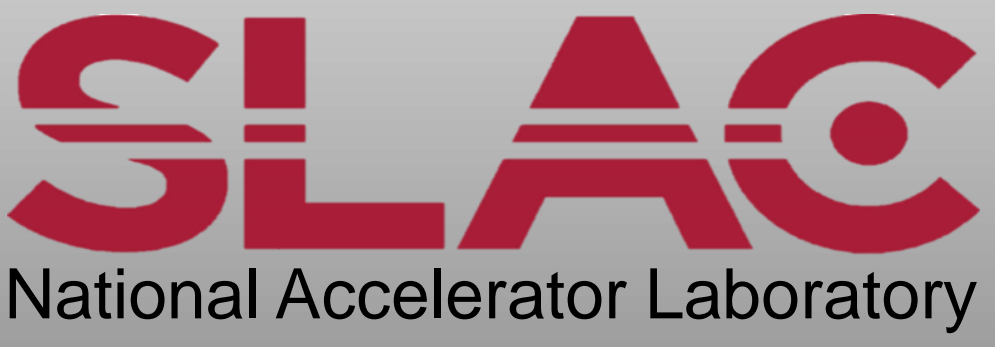

You

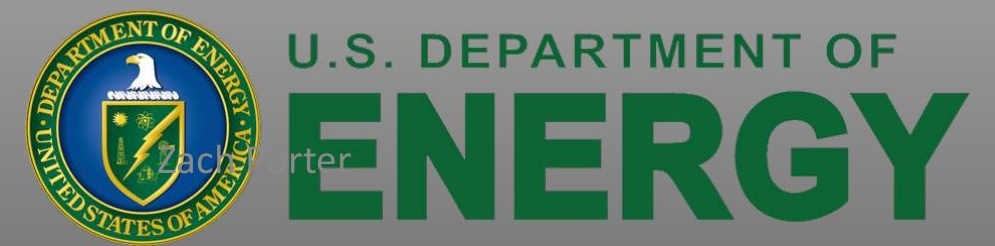

Office of

Science 